\title{
XLV. On the application of the theory of chemical potential to the thermodynamical theory of solutions
}

\section{S.A. Shorter B.Sc.}

To cite this article: S.A. Shorter B.Sc. (1912) XLV. On the application of the theory of chemical potential to the thermodynamical theory of solutions, Philosophical Magazine Series 6, 23:136, 483-494, DOI: $10.1080 / 14786440408637244$

To link to this article: http://dx.doi.org/10.1080/14786440408637244

冓 Published online: 20 Apr 2009.

Submit your article to this journal $[\pi$

Џll Article views: 2

Q View related articles ๘

7 Citing articles: 1 View citing articles $\square$ 
preted is described, and the results brought to such a form that they can be compared with theory.

16-18. The results are discussed first with reference to the theory of ionization by collision. It is shown that they afford considerable support for it, if the first of the two alternative theories as to the nature of the negative ion put forward in the previous paper is adopted. They do not seem to agree with the second theory. It is concluded that in dry air an electron remains an electron through most of its career, and that the formation of complex negative ions can only occur rarely if at all.

18. The conclusions to be drawn from the results in the matter of the speed of the delta rays is considered. It is shown that they support the view that the initial speed is small compared with 20 volts.

19. The conclusions concerning delta rays which have been attained in this and two preceding papers are discussed with a view to the light that they throw upon the mechanism of ionization.

It is, of course, clear that the brief discussion given of the mechanism of ionization is largely due to the suggestions of Prof. Bragg, to whose inspiration all this work is due.

Leeds University, Nov. 18, 1911.

XLV. On the Application of the Theory of Chemical Potential to the Thermodynamical Theory of Solutions. By S. A. Shorter, B.Sc., Assistant Lecturer in Physics in the University of Leeds.-Part II. The Effect of Pressure on Vapour-Pressure. The Vapour-Pressure Theory of Osmotic Pressure. The Freezing of Solutions *

$\mathrm{T} \mathrm{N}$ a previous communication $\dagger$ we considered the equilibrium between the solution and solvent vapour in the case where the surface of separation is plane, and the pressure, therefore, the same in both phases. If the surface of separation is curved, the pressures in the two phases will differ by an amount equal to the product of the surface tension and the total curvature, and the conditions of equilibrium will, of course, be modified. We may also suppose this co-existence of the two phases to be brought about by means of an insoluble gas. 'This involves the introduction of the idea of partial pressure, as well as the assumption of

* Communicated by the Author.

+ Phil. Mag. xxii. p. 933, Dec. 1911. The author takes this opportunity of making the following corrections in Part I. :- page 934, line 17 from top, for $S$ read $s$ : page 938 , line 6 from bottom, for $P_{0}\left(s, p_{0}, \theta\right)$ read $\mathrm{P}_{v}(s, p, \theta)$. 
the existence of an insoluble gas. We will therefore confine ourselves to the consideration of the effect of capillary pressure on the vapour-pressure of a solution.

We will also consider the thermodynamical theory of the equilibrum between the solution and solid solvent, and establish exact formulæ connecting the freezing-point with the osmotic pressure and vapour-pressure. These formulæe, which are obviously of fundamental importauce in the theory of solutions, appear never to have been previously established.

\section{Some new Symbols used in this Communication.}

The following new symbols will be used in the part of the paper dealing with the freezing of solutions. The new symbols used in the earlier parts of the paper will be defined as they occur in the text.

$T_{0}$ the freezing-point of the pure solvent under a

T the freezing - point of the solution under a pressure $p$;

$\mathrm{I}_{0}$ the latent hent of fusion of the solid solvent, when in equilibrium with the pure liquid solvent under a pressure $p^{*}$;

$\gamma(s, p, \tau)$ the specific heat of the solution under a constant pressure $p$, and at a temperature $\tau$;

$\gamma_{0}(p, \tau)$ the specific heat (under constant pressure) of the pure liquid solvent;

$\gamma_{s}(p, \tau)$ the specific heat (under constant pressure) of the solid solvent;

$\gamma_{d}(p, \tau) \equiv \gamma_{0}(p, \tau)-\gamma_{d}(p, \tau)$;

$l_{0}(s, p, \theta)$ the heat of dilution at a pressure $p$ and temperature $\theta \dagger$.

$\phi_{0}(p, \theta)$ the chemical potential of the solid solvent at at pressare $p$ and temperature $\theta$;

$\Delta_{0}(s, p, \theta) \equiv f_{0}(0, p, \theta)-f_{0}(s, p, \theta)$;

$\Gamma_{0}(p, \theta)=\frac{\partial}{\partial \theta} \phi_{0}(p, \theta)-\frac{\partial}{\partial \theta} f_{0}(0, p, \theta)$;

$\delta_{0}(s, p, \theta) \equiv \frac{\partial}{\partial p} \Delta_{0}(s, p, \theta)$.

* It is unnecessary to adopt the more precise notation $\mathrm{T}_{0}(p), \mathrm{T}(s, p)$, $\mathrm{L}_{0}(p)$, as we shall not consider variations of $s$ and $p$.

$\uparrow$ The heat evolved when an infinitesimal mass $\delta \mathrm{M}_{0}$ of the solvent is adder to the solution (pressure and temperature being maintained constant $)$ is $l_{0}(s, p, \theta) \delta \mathrm{M}_{0}$. 
Dashes will be used to indicate differentiation with respect to the temperature, thus :

$$
l_{0}^{\prime}(s, p, \theta)=\frac{\partial}{\partial \theta} l_{v}(s, p, \theta), \phi_{0}{ }^{\prime \prime}(p, \theta)=\frac{\partial^{2}}{\partial \theta^{2}} \phi_{v}(p, \theta) .
$$

\section{The Effect of Pressure on Vapour-Pressure.}

If the solution and solvent vapour are in equilibrium, the surface of separation latving a curvature such that the pressure of the solution exceeds that of the vapour by an amount $\gamma_{c}$, the vapour-pressure $\Pi_{c}$ is determined by the equation

$$
f_{0}\left(s, \Pi_{c}+p_{c}^{s}, \theta\right)=\mathrm{F}_{0}\left(\Pi_{c}, \theta\right) .
$$

When the surface of separation is plane the vapour-pressure II is determined by the equation

$$
f_{0}(s, \Pi, \theta)=\mathrm{F}_{0}(\Pi, \theta) \text {. }
$$

Hence we have

$$
\left(\Pi_{c}+p_{c}-\Pi\right) \mathrm{P}_{v}\left(s, \Pi \rightarrow \Pi_{c}+p_{c}, \theta\right)=\int_{\Pi}^{\Pi_{c}} V(x, \theta) d x . .
$$

This equation agrees with that obtained in a different manner by Callendar * for the effect of capillary pressure, and with that obtained by Porter $\dagger$ for the effect of the pressure of an insoluble gas.

In the case of the pure solvent, the ordinary vapourpressure $\Pi_{0}$ and the vapour-pressure $\Pi_{0 c}$ corresponding to a capillary excess pressure $p_{c}$, are related by the following equation, which may be derived from equation (1) by putting $s=0$,

$$
\left(\Pi_{0 c}+p_{c}-\Pi_{v}\right) v\left(0, \Pi_{0} \rightarrow \Pi_{0 c}+p_{c}, \theta\right)=\int_{\Pi_{0}}^{\Pi_{0 s}} \mathrm{~V}(x, \theta) d x .
$$

If we neglect the compressibility of the liquid and suppose that the solvent rapour behaves as an ideal gas, we obtain the well-known expression $\ddagger$ for the effect of capiliary curvature on the vapour-pressure of a liquid.

* Roy. Soc. Proc. A. vol. 1xxx. p. 466 (1907).

† Roy. Soc. Proc. A. vol. Ixxix. p. 519 (1907).

$\ddagger$ J. J. Thomson, 'Conduction of Electricity through Gases,' p. 180. 


\section{Generalised Vapour-Pressure and the Vapour-P'ressure} Theory of Osmotic Pressure.

Under ordinary circumstances the solution and solvent vapour when in equilibrium are under the same pressure, so that the vapour-pressure is a function of the concentration and temperature. We have seen, however, that the two phases may co-exist under different pressures. We may therefore generalise the idea of vapour-pressure, and suppose a solution of given concentration at a given temperature to have different vapour-pressures corresponding to different pressures in the solution. The generalised vapour-pressure P of a solution is determined as a function of the concentration, temperature, and pressure in the solution, by the equation

$$
f_{0}(s, p, \theta)=\mathrm{F}_{\mathbf{o}}(\mathbf{P}, \theta) \text {. . . . . }
$$

If we differentiate this equation with respect to the solution pressure, we obtain the equation

$$
\frac{\partial \mathbf{P}}{\partial p}=\frac{\mathrm{P}_{\mathrm{v}}(s, p, \theta)}{\mathrm{V}(\mathbf{P}, \boldsymbol{\theta})} \quad \cdots . \cdot .
$$

showing the effect of pressure on the generalised vapourpressure.

Suppose that we have a solution of concentration $s_{1}$ under a pressure $p_{1}$ in osmotic equilibrium with a solution of concentration $s_{2}$ under a pressure $p_{2}$. The generalised vapourpressures $\mathbf{P}_{1}$ and $\mathbf{P}_{2}$ of the two solutions are determined by the equations

$$
\begin{aligned}
& f_{0}\left(s_{1}, p_{1}, \boldsymbol{\theta}\right)=\mathrm{F}_{\theta}\left(\mathbf{P}_{1}, \theta\right), \\
& \dot{f}_{0}\left(s_{2}, p_{2}, \theta\right)=\mathrm{F}_{0}\left(\mathbf{P}_{2}, \boldsymbol{\theta}\right) .
\end{aligned}
$$

Since the solutions are in osmotic equilibrium we must have

$$
f_{0}\left(s_{1}, p_{1}, \theta\right)=f_{0}\left(s_{2}, p_{2}, \theta\right) \text {. }
$$

Hence we have

$$
\mathrm{F}_{0}\left(\mathbf{P}_{1}, \theta\right)=\mathrm{F}_{0}\left(\mathbf{P}_{2}, \theta\right) ;
$$

and therefore, since $F_{0}$ always increases with the pressure,

$$
\mathbf{P}_{1}=\mathbf{P}_{2} \text {. }
$$

Hence two solutions in osmotic equilibrium have the same generalised vapour-pressure. The converse of this is obviously true. Two solutions of different concentrations under pressures such that their generalised vapour-pressures are equal will be in osmotic equilibrium under these pressures.

We may therefore regard the osmotic difference of 
pressure as the increase of pressure which must be applied to the stronger solution to make its generalised vapour-pressure equal to that of the weaker solution. It seems to me, however, that there is nothing fundamental in this idea. It seems quite a mistake to define the osmotic pressure of a solution as the increase of pressure which must be applied to the solution to make its vapour-pressure equal to that of the pure solvent. 'Ihus Callendar's * vapour-pressure theory of osmotic pressure "according to which the osmotic pressure is simply the pressure required to produce equilibrium of vapour-pressure between the solvent and solution" is merely a particular statement of the general thermodynamical truism that the conditions of co-existence of two parts of a system are independent of the precise nature of the mechanism of the co-existence, and depend only on the "constraints" of the mechanism. Callendar's "vapour-sieve" has the same constraint as the semi-permeable membrane-it does not allow the solute to pass from one part of the system to the other. Hence the conditions of equilibrium are the same in the two cases. Whatever may be the nature of the osmotic mechanism, and whatever may be the relation between the magnitude of the osmotic pressure and the composition of the solution, the statement quoted above is true; so it can in no sense be regarded as a theory of osmotic pressure.

\section{The Thermodynamical theory of the Equilibrium between the Solution and Solid Solvent.}

If the pure liquid solvent is in equilibrium with the solid solvent, the temperature $\mathrm{T}_{0}$ is determined as a function of the pressure only, by the equation

$$
f_{0}\left(0, p, \mathrm{~T}_{0}\right)=\phi_{0}\left(p, \mathrm{~T}_{0}\right) \text {. . . . . }
$$

If the solution is in equilibrium with the solid solvent, the temperature $\mathrm{T}$ is determined as a function of the concentration and pressure by the equation

$$
f_{0}(s, p, \mathrm{~T})=\phi_{0}(p, \mathrm{~T}) \cdot . \quad \cdot \quad \cdot
$$

Freezing-point determination forms a third method of investigating the thermodynamical properties of solutions. In order to compare the results of such determinations with measurements of the osmotic pressure and vapour-pressure, it is necessary to obtain an expression for the lowering of the chemical potential of the solvent caused by the addition of the solute, similar to those which have been obtained in 
488 Mr. S. A. Shorter : Application of the Theory

the cases of osmatic pressure (equation (5) of Part I.) and vapour-pressure (equation (8) of Part I.). The process of obtaining an exact formula is more complicated in the present case than in the two other cases, because we have to deal with variations of temperature, and the temperature derivative of the chemical potential is not connected with quantities which may be determined experimentally in such a simple manner as the pressure derivative.

We will first calculate the lowering of the solvent potential at the freezing-point of the solution. From equations (5) and (6) we obtain

$$
f_{0}\left(0, p, \mathrm{~T}_{0}\right)-f_{0}(s, p, \mathrm{~T})=\int_{\mathrm{T}}^{\mathrm{T}_{0}} \phi_{0}{ }^{\prime}(p, \theta) d \theta
$$

but

$$
f_{0}\left(0, p, \mathrm{~T}_{0}\right)=f_{0}(0, p, \mathrm{~T})+\int_{\mathrm{T}}^{\mathrm{T}_{0}} f_{0}^{\prime}(0, p, \theta) d \theta
$$

so that

$$
\Delta_{0}(s, p, \mathrm{~T})=\int_{\mathbf{T}}^{\mathrm{T}_{\varphi}} \Gamma_{0}(p, \theta) d \theta . . \cdot
$$

The value of $\Gamma_{0}$ is known for one value of the temperature, viz., the value $\mathrm{T}_{0}$. We have the well-known relation*

$$
\Gamma_{0}\left(p, \mathrm{~T}_{0}\right)=\frac{\mathrm{L}_{0}}{\mathrm{~T}_{0}^{-}} \text {. }
$$

The value of the temperature derivative of $\Gamma_{0}$ is known for any value of the temperature. We have the well-known relations $\dagger$

so that

$$
\begin{aligned}
\tau \phi_{0}{ }^{\prime \prime}(p, \tau) & =-\gamma_{s}(p, \tau), \\
\tau f_{0}^{\prime \prime}(0, p, \tau) & =-\gamma_{0}(p, \tau),
\end{aligned}
$$

$$
\Gamma_{0}^{\prime}(p, \tau)=\frac{\gamma_{d}(p, \tau)}{\tau} \text {. }
$$

The value of $\Gamma_{0}$ at any temperature $\theta$, between $\mathrm{T}$ and $\mathrm{T}_{0}$, is therefore given by the equation

$$
\Gamma_{0}(p, \theta)=\frac{\mathrm{L}_{0}}{\mathrm{~T}_{0}}-\int_{\theta}^{\mathrm{T}_{0}} \frac{\gamma_{d}(p, \tau)}{\tau} d \tau
$$

* Duhem, La Mécanique Chimique, vol. ii. pp. 5 and 54; Preston, s Theory of Heat,' p. 776 (second edition).

+ Duhem, loc, cit. rol. i. p. 110; Jreston, loc. cit. p. 770. 
Substititing this ralue in equation (7), and performing the integration with respect to $\theta$, we oltain the following expression for the lowering of the solvent potential at the freezing-point of the solution :-

$\Delta_{0}(s, p, \mathrm{~T})=\frac{\mathrm{J}_{0}\left(\mathrm{~T}_{0}-\mathrm{T}\right)}{\mathrm{I}_{0}}-\int_{\mathrm{T}}^{\mathrm{T}_{0}} \gamma_{d}(p, \tau) d \tau+\mathrm{T} \int_{\mathrm{T}}^{\mathrm{T}_{0}} \frac{\gamma_{d}(p, \tau)}{\tau} d \tau$.

The osmotic and vapour-pressnre measurements with which the freezing-point determinations have to be compared may have been made at any temperature. It is necessary, therefore, to obtain a formula connecting the potential lowering at two different temperatures. Such a formula is also usetul for the comparison of osmotic and vapour-pressure measurements made at different temperatures. We will therefore consider this question in a general manner (apart from its present application) in the next section.

\section{The Variation of the Solvent Potential Louering with Temperature.}

Duhem's expression * for the heat of dilution in terms of the solvent potential lowering may be written in the form

$$
\partial \theta\left\{\frac{\Delta_{0}(s, p, \theta)}{\theta}\right\}=-\frac{l_{0}(s, p, \theta)}{\theta^{2}} . . .
$$

Hence if $\theta_{1}$ and $\theta_{2}$ are any two temperatures we have

$$
\frac{\Delta_{0}\left(s, p, \theta_{2}\right)}{\theta_{2}}-\frac{\Delta_{0}\left(s, p, \theta_{1}\right)}{{ }_{-}}=-\int_{\theta_{1}}^{\theta_{2}} \frac{l_{0}(s, p, \theta)}{\theta^{2}} d \theta .
$$

By combining this equation with equation (8) we could obtain an expression connecting the potential lowering at any temperature with the freezing-point. The equation involves, however, a knowledge of the heat of dilution as a function of the temperature. Now in general the heat of dilution will be known at one temperature only. We will therefore deduce a formula involving a knowledge of the heat of dilution for a single value of the temperature, making use of the well-known expression connecting the temperature derivative of the heat of dilution with specitic heat data :

$$
\begin{gathered}
l_{0}^{\prime}(s, p, \theta)=\gamma_{0}(p, \theta)-\gamma(s, p, \theta)+s(1+s) \frac{\partial}{\partial s} \gamma(s, p, \theta) \cdot(11) \\
\text { * Loc. cit. vol. iij. p. } 132 .
\end{gathered}
$$


The value of the heat of dilution at any temperature $\theta$ is given, in terms of its value at the temperature $\theta_{0}$, by

$$
l_{0}(s, p, \theta)=l_{\mathrm{u}}\left(s, p, \theta_{0}\right)-\int_{\theta}^{\theta_{0}} l_{0}^{\prime}(s, p, \tau) d \tau .
$$

Substituting this value in equation (10) and performing the integration with respect to $\theta$, we obtain

$$
\begin{aligned}
\frac{\Delta_{0}\left(s, p, \theta_{2}\right)}{\theta_{2}} & -\frac{\Delta_{0}\left(s, p, \theta_{1}\right)}{\theta_{1}} \\
= & -l_{0}\left(s, p, \theta_{0}\right)\left(\frac{1}{\theta_{1}}-\frac{1}{\theta_{2}}\right)+\frac{1}{\theta_{1_{2}}} \int_{\theta_{1}}^{\theta_{0}} l_{0}^{\prime}(s, p, \tau) d \tau \\
& -\frac{1}{\theta_{2}} \int_{\theta_{2}}^{\theta_{0}} l_{0}^{\prime}(s, p, \tau) d \tau-\int_{\theta_{1}}^{\theta_{2}} \frac{l_{0}^{\prime}(s, p, \tau)}{\tau} d \tau .
\end{aligned}
$$

This exact formula connecting the values of the solvent potential lowering at two different temperatures may be simplified if we assume that the specific heats involved are independent of the temperature. The equation then reduces to

$$
\begin{aligned}
\frac{\Delta_{0}\left(s, p, \theta_{2}\right)}{\theta_{2}}-\frac{\Delta_{n}\left(s, p, \theta_{1}\right)}{\theta_{1}}= & -l_{0}\left(s, p, \theta_{0}\right)\left(\frac{1}{\theta_{1}}-\frac{1}{\theta_{2}}\right) \\
& +l_{0}^{\prime}\left\{\frac{\theta_{0}\left(\theta_{2}-\theta_{1}\right)}{\theta_{1} \theta_{2}}-\log _{\theta_{1}}^{\theta_{2}}\right\} .
\end{aligned}
$$

For the purpose of practical calculation the coefficient of $l_{0}^{\prime}$ may be written in the form

$$
\frac{\theta_{2}-\theta_{1}}{\theta_{1}}\left\{\frac{\theta_{0}-\bar{\theta}}{\theta_{2}}+\frac{1}{6}\left(\frac{\theta_{2}-\theta_{1}}{\theta_{1}}\right)^{2}-\frac{1}{4}\left(\frac{\theta_{2}-\theta_{1}}{\theta_{1}}\right)^{3}+\ldots\right\},
$$

where

$$
\bar{\theta}=\frac{\theta_{1}+\theta_{2}}{2} \text {. }
$$

\section{The Relation between the Osmotic Pressure and \\ Vapour-Pressure and the Freezing-Point.}

Equations (5) and (8) of Part I. give the value of the solvent potential lowering in terms of the osmotic pressure and vapour-pressure respectively. These two formulæ involve different values of the pressure, but the effect of variations of pressure is easily calculated. Adopting a notation for the mean value of $\delta_{0}$ similar to that adopted in the case of the function $\mathrm{P}_{0}$, we have, if $p_{1}$ and $p_{2}$ are two 
values of the pressure,

$$
\Delta_{0}\left(s, p_{2}, \theta\right)-\Delta_{0}\left(s, p_{1}, \theta\right)=\left(p_{2}-p_{1}\right) \delta_{0}\left(s, p_{1} \rightarrow p_{2}, \theta\right) .
$$

The mean value of $\delta_{0}$ is easily calculated. In general, however, the effect of pressure variations is so small that the value of $\delta_{0}$ corresponding to atmospheric pressure may be used in this formula.

The value, at a pressure $p$ and temperalure $t$, of the solvent potential lowering is given, therefore, in terms of the osmotic pressure at the same temperature, corresponding to a pure solvent pressure $p_{0}$, by the equation

$$
\begin{array}{r}
\Delta_{0}(s, p, t)=\Omega\left(s, p_{0}, t\right) \mathrm{P}_{0}\left(s, p_{0} \rightarrow p_{0}+\Omega, t\right) \\
+\left(p-p_{0}\right) \delta_{0}\left(s, p_{0} \rightarrow p, t\right), . .
\end{array}
$$

and in terms of the vapour-pressure at the same temperature, by the equation

$$
\begin{array}{r}
\Delta_{0}(s, p, t)=\int_{\mathrm{II}}^{\Pi_{0}} \mathrm{~V}(x, t) d x-\left(\Pi_{0}-\Pi\right) \mathrm{P}_{0}\left(s, \Pi \rightarrow \Pi_{0}, t\right) \\
+\left(p-\Pi_{0}\right) \delta_{0}\left(s, \Pi_{0} \rightarrow p, t\right) .
\end{array}
$$

Equation (8) gives the value of the solvent potential lowering at the freezing-point of the solution. Calculating the value at the temperature $t$ by means of equation (12) we obtain the formula

$$
\begin{aligned}
\Delta_{0}(s, p, t)= & \mathrm{L}_{0} t\left(\frac{1}{\mathrm{~T}}-\frac{1}{\mathrm{~T}_{0}^{\prime}}\right)-\frac{t-\mathrm{T}}{\mathrm{T}} l_{0}\left(s, p, \theta_{0}\right)-\frac{t}{\mathrm{~T}} \int_{\mathrm{T}}^{\mathrm{T}_{0}} \gamma d(p, \tau) d \tau \\
& +t \int_{\mathrm{T}}^{\mathrm{T}_{0}} \frac{\gamma_{d}(p, \tau)}{\tau} d \tau+\frac{t}{\mathrm{~T}} \int_{\mathrm{T}}^{\theta_{0}} l_{0}^{\prime}(s, p, \tau) d \tau \\
& -\int_{t}^{\theta_{0}} l_{0}^{\prime}(s, p, \tau) d \tau-t \int_{\mathrm{T}}^{t} \frac{l_{0}^{\prime}(s, p, \tau)}{\tau} d \tau . \quad \text { (17) }
\end{aligned}
$$

Fiquations (15) and (17) give an exact relation between the osmotic pressure and the freezing-point, and equations (16) and (17) an exact relation between the vapour-pressure and the freezing-point. By combining equations (15) and (16) we obtain the equation connecting vapour-pressure and osmoțic pręssure deduced in a slightly different manner in Part I.

In the next section we will simplify these relations by making various approximations and compare the results thus obtained with expressions deduced by other investigators. 


\section{Mr. S. A. Shorter : Application of the Theory}

Approximate Fipressions connecting the Freezing-Point with the Solvent Potential Lowering, Osmotic Pressure, and

Vapour-Pressure.

If we remard all the specific heats involved in equation (17) as independent of the temperature, the equation rednces to the simpler form

$$
\begin{aligned}
\Delta_{0}(s, p, t)=\mathrm{L}_{0} t\left(\frac{1}{\mathrm{~T}}-\frac{1}{\mathrm{~T}_{0}}\right) & -\frac{t-\mathrm{T}}{\mathrm{T}} l_{0}\left(s, p, \theta_{0}\right)-\gamma_{d} t\left(\frac{\mathrm{T}_{0}-\mathrm{T}}{\mathrm{T}}-\log \frac{\mathrm{T}_{0}}{\mathrm{~T}^{\mathrm{i}}}\right) \\
& +l_{0}^{\prime} t\left\{\frac{\theta_{0}(t-\mathrm{T})}{\mathrm{T} t}-\log _{\frac{t}{\mathrm{~T}}}\right\} . \cdot(18)
\end{aligned}
$$

For the purpose of practical calculation, the equation may be writen in the form

$$
\begin{aligned}
\Delta_{0}(s, p, t)= & \mathrm{L}_{0} t\left(\frac{1}{\mathrm{~L}^{\prime}}-\frac{1}{\mathrm{~T}}\right)-\frac{t-\mathrm{T}}{\mathrm{T}} l_{0}\left(s, p, \theta_{0}\right) \\
& -\gamma_{d} t\left\{\frac{1}{2}\left(\frac{\mathrm{T}_{0}-\mathrm{T}}{\mathrm{T}}\right)^{2}-\frac{1}{3}\left(\frac{\mathrm{T}_{0}-\mathrm{T}}{\mathrm{T}}\right)^{3}+\ldots\right\} \\
& +\frac{l_{0} t(t-\mathrm{T})}{\mathrm{T}}\left\{\frac{\theta_{0}-\bar{\theta}}{t}+\frac{1}{6}\left(\frac{t-\mathrm{T}}{\mathrm{T}}\right)^{2}-\ldots\right\}, .
\end{aligned}
$$

where

$$
\bar{\theta}=\frac{t+\mathrm{T}}{2}
$$

The relation between the osmotic pressure and the freezingpoint may be simplified in the following manner. Let us suppose that the pure solvent in the case of the osmotic pressure measurements, and the solution in the case of the freezing-point determinations, are both under atmospheric pressure. We may express the mean value of $P_{0}$ in terms of its value at atmospheric pressure by means of equation (2) of Part I. Simplifying equation (15) in this manner and combining it with equation (19) we obtain the following relation $^{*}:-$

$$
\begin{aligned}
& \Omega(s, \varpi, t) \mathrm{P}_{0}(s, \varpi, t)\left(1-\frac{1}{2} \lambda \Omega\right)=\mathrm{L}_{0} t\left(\frac{1}{\mathrm{~T}}-\frac{1}{\mathrm{~T}_{0}}\right) \\
& \quad-\frac{t-\mathrm{T}}{\mathrm{T}} l_{0}\left(s, \varpi, \theta_{0}\right)-\gamma_{d} t\left\{\frac{1}{2}\left(\frac{\mathrm{T}_{0}-\mathrm{T}}{\mathrm{T}}\right)^{2}-\frac{1}{3}\left(\frac{\mathrm{T}_{0}-\mathrm{T}}{\mathrm{T}}\right)^{3}+\ldots\right\} \\
& \quad+\frac{l_{0} t(t-\mathrm{T})}{\mathrm{T}}\left\{\frac{\theta_{0}-\bar{\theta}}{t}+\frac{1}{6}\left(\frac{t-\mathrm{T}}{\mathrm{T}}\right)^{2}-\ldots\right\} \cdot \cdot \cdot \cdot(20)
\end{aligned}
$$

* It is quite justifiable to take account of the compressibility and at the same time neglect the variation with temperature of the specific heats. 
If in the above equation we make $\theta_{0}=t$, and neglect the compressibility and powers of $\mathrm{T}_{0}-\mathrm{T}$ and $t-\mathrm{T}$ higher than the second, we obtain Ewan's expression * connecting the osmotic pressure and the freering-point.

In the absence of any information regarding the precise form of the isothermal of the solvent vapour, the definite integral in equation (16) must be evaluated on the assumption that the solvent vapour behaves as an ideal gas, $i$. e., we must assume that

$$
\int_{\Pi}^{\Pi_{0}} \mathrm{~V}(x, t) d x=\mathrm{R}_{0} t \log \frac{\Pi_{0}}{\Pi}, \ldots . .
$$

where $R_{0}$ is the "gas constant" of the solvent vaponr. If we make this assumption we must, of course, neglect the term $\left(\Pi_{0}-\Pi\right) \mathrm{P}_{0}$. The magnitude of the third term on the right-hand side of equation (16) will depend upon several factors. Speaking generally, we may say that its retention in the equation is justifiable only in the case of strong solutions at low temperatures (when $\delta_{0}$ is a fairly large fraction of the specific volume of the pure solvent, and $\Pi_{0}-\Pi$ only a small fraction of $p-\Pi_{0}$ ). Making these approximations and putting $p=\sigma$, we obtain the following expression connecting the vapour-pressure and the freezing-point :-

$$
\begin{aligned}
\mathrm{R}_{v} t & \log \frac{\Pi_{0}}{\Pi}+\left(\varpi-\Pi_{0}\right) \delta_{0}(s, \varpi, t)=\mathrm{L}_{0} t\left(\frac{1}{\mathrm{~T}}-\frac{1}{\mathrm{~T}_{0}}\right) \\
& -\frac{t-\mathrm{T}}{\mathrm{T}} l_{0}\left(s, \varpi, \theta_{0}\right)-\gamma_{d} t\left\{\frac{1}{2}\left(\frac{\mathrm{T}_{0}-\mathrm{T}}{\mathrm{T}}\right)^{2}-\frac{1}{3}\left(\begin{array}{c}
\mathrm{T}_{0}-\mathrm{T} \\
\mathrm{T}
\end{array}\right)^{3}+\ldots\right\} \\
& +\frac{l_{0} t(t-\mathrm{T})}{\mathrm{T}}\left\{\frac{\theta_{0}-\bar{\theta}}{t}+\frac{1}{6}\left(\frac{t-\mathrm{T}}{t}\right)^{2}-\ldots\right\} . . .
\end{aligned}
$$

If we make $t=\mathrm{T}_{0}$, and neglect the terms containing $\delta_{0}$ and $l_{0}^{\prime}$, we obtain Dieterici's $\dagger$ equation connecting the freezing-point of the solution with the vapour-pressure at the pure solvent freezing-point.

If we substitute in equation (8) the approximate value of the potential lowering calculated as in equation (21) we obtain Callendar's $\ddagger$ expression for the ratio of the vapourpressures of the pure solvent and solution at the freezingpoint of the latter.

The simplest method of comparing a large number of experimental data relating to a particular solution is to calculate the value of the solvent potential lowering at some

* Zeit. fiir Phys. Chem. xxxi. p. 22 (1899).

+ Wied. Ann. lii. p. 263 (1894). 
standard pressure and temperature. By means of the formulæ established in this work this may be done to a degree of accuracy limited only by the accuracy of the experimental data. The writer hopes to publish some examples of such a comparison at a later date.

The third (and last) part of the present work will deal with the effect of gravity on a solution, and the extension of the theory to the case of a solution containing any number of involatile solutes.

The University, Leeds, Dec, 23, 1911.

XLVI. A New Approach to the Theory of Relativity. By Einward V. Huntington, Ph.D., Assistant Professor of Mathematics in Harvard University *.

INTRODUCTION.

I. System at Rest in the Aither.

The regulating and setting of stationary clocks.

Laying out a system of coordinates by light signals.

Definition of distance.

Definition of observed velocity.

Definition of observed rate of a moving clock.

Doppler's principle.

II. System in Unfform Motion throdgh the Ether.

The transformation equations.

Features of the moving system.

The composition of velocities.

III. The Principle of Relativity.

The inverse transformation equations.

Experiments in which no observer leaves his own station.

Experiments with a portable measuring-rod; first physical assumption involved in the theory of relativity.

Experiments with a portable clock ; second physical assumption involved in the theory of relativity.

Appendix.

\section{INTRODUC'TION.}

TWE Theory of Relativity as developed by Einstein $\dagger$ is usually supposed to involve a radical modification, not only of our conception of the ather, but also of our fundamental notions concerning space and time; and the discussion of the so-called "paradoxes of relativity" has

* Communicated by the Author. Reprinted from the Heinrich Weber Festschrift (Teubner, 1912).

† A. Einstein, "Zur elektrodynımik bewegter Körper," Annalen der Plysil, ser. 4, vol. xvii. pp. $891-921$ (1905). 